\title{
Sun-to-Heel Energy Efficiency of Transportation Fuels
}

\author{
Jia Yong Leo, and Ghim Ping Ong
}

\begin{abstract}
Life cycle analysis (LCA) is a technique to assess the environmental impact of a product or service throughout its life cycle and is used commonly to evaluate transport decisions at the planning level. Well-to-Wheel (WtW) is a subset of LCA that focuses on the efficiency of transport fuels by measuring fuel usage, greenhouse gas emissions and other pollutants for a given pathway from the oil wells to the wheels of a vehicle being studied. The method has been commonly used to evaluate environmentally-friendly transport projects or applications. However, such methods do not consider the first and last mile connection and hence could not be adopted for planning-related transport applications (for example, transit-oriented development). This paper proposes a new approach of LCA - sun-to-heel (StH) method of LCA where a holistic system wide perspective based on trips, including first and last mile connections are considered during the environmental impact assessment of a transport product or service. Using secondary data collected from various transport applications, it can be shown that the StH method is a much more comprehensive approach in evaluating environmental impact of transitfriendly transportation innovations as compared to the WtW method.
\end{abstract}

Index Terms - life cycle analysis, sun-to-heel, transport energy, efficiency, well-to-wheel.

\section{INTRODUCTION}

$\mathrm{L}$ ife cycle assessments (LCA) are commonly used to analysis the environmental impacts of products or services, for example the amount of water used to make every pair of jeans or the amount of greenhouse gas emission for a transatlantic flight. Wheel-to-wheel (WtW)) is a subset of the LCA methodology that focuses on the energy efficiency of transportation fuels. WtW provides analysis into the amount of energy used and various emissions emitted throughout the supply chain of the fuel of the vehicle, from the extraction phase, processing, distribution, and combustion in the engines. However, this focus on the vehicles and fuel pathways misses the fact that transportation is a service, and the commuter's

J.Y. Leong is with the School of Design and Environment, National University of Singapore, Singapore. ceeongr@nus.edu.sg (e-mail: ceeongr@nus.edu.sg)

G. P. Ong is with the Department of Civil and Environmental Engineering , National University of Singapore, Singapore and School of Design and Environment, National University of Singapore, Singapore. ceeongr@nus.edu.sg. objective is to reach their destination, and not the distance travelled.

This paper will introduce a new method of accounting for transportation's cost to the planet's energy reserves, built on the LCA and WtW methodologies that puts the objective of the commuter back in focus, completing the trip. The concept of sun-to-heel $(\mathrm{StH})$ will be introduced by bringing the central elements that $\mathrm{StH}$ is concerned about into the discussion. Namely these are the commuters, the environment they exist in, the city, the resource they ultimately draw on for their activities, Primary Energy. The StH methodology will then be discussed in detail.

\section{Sun-To-HeEl CONCEPT}

The sun-to-heel metric is the measurement of the amount of primary energy that is accountable to a commuting journey, or trip. In essence, this metric is a measure of how much the commuter takes from the planet to get from their origin to destination in a city. The following subsections shall describe the basic elements of the sun-to-heel concept.

\section{A. The commuter}

The commuter is someone that is concerned about, and in the act of getting from Point A to Point B. Individually, the concerns of the commuter span from routine travelling to and from work, social reasons of meeting up with family and friends, or as tourists getting around town. The reason why someone travels from Point A to Point B is simply because what that person needs at that time is not available at Point A, and is available at Point B. For example, many commuters make their daily commute to the work place because employment is not found at their homes or origin zones, but at the workplace or destination zones.

\section{B. The city}

Commuting is a vital service provided by the city to its inhabitants that is vital to economic activity, and a basic human need. In order to provide this service, land and capital has been set aside for transportation networks, roads, rails, ports, airports and others. Energy is consumed daily within the city when commuters travel. In addition, commuting costs commuters time and money. It also costs the city opportunities, capital and space that could be better utilised for other purposes, long commutes also reduce the quality of life in a city. In today's context, where the main fuel source, particularly for vehicles, has a non-renewable source, and a sink for bi-products that has already overshot its carrying capacity, energy efficiency needs to be key concern. By some 
estimates, energy efficiency needs to triple in order to stay within $2^{\circ} \mathrm{C}$ of warming [1].

A trip, i.e. a commute from Point A to Point B, is chosen as the denominator of this metric as it filters out red herrings like the efficiency of a particular engine and focuses on the amount of energy needed to get a commuter from point to point. The fact that distance is not used as a denominator is a central feature of this metric. Using trips as the denominator will give credit to journeys that are short, made using energy efficient vehicles, which are powered by an efficient energy supply chain. This is only possible with good city planning, efficient power infrastructure, and policy that encourages adoption of energy efficient technologies.

When it comes to energy efficiency in transportation, much of the focus has been on the efficiency of the vehicles. Models such as Argonne National Laboratory's GREET model [2] focuses on the well-to-wheel energy requirements of transportation. However, by taking the view of the bigger picture, time, energy, commuter discomfort, all can be minimized if the distance that needs to be travelled is also minimised. This means that the city plan, the urban fabric, plays a big role in the energy efficiency of the transportation needs of a city, along with the technologies deployed in the transportation and energy sectors.

\section{Primary energy}

Primary energy is energy that has not been converted or transformed that are found in nature, for example fossil fuels, wind energy, solar radiation, geothermal, nuclear fuels, potential energy stored by water behind dams and others [3]. The amount of primary energy needed to perform work will always be greater than the energy converted to perform said work. This is due to conversion, transmission, transport losses between the source and the point of use.

\section{Primary energy of fossil fuels and renewables}

The primary energy contained within a fossil fuel, or its derivative fuel products, is the caloric value it contains. For sources of energy where there is no combustion involved, namely nuclear, geothermal, wind, photovoltaic solar, and thermal solar primary energy is defined differently. A common boundary has been defined as "the first flow in the production process that has a practical energy use" [4]. Specifically, this means that heat generated during combustion for combustible fuels, heat for nuclear, solar thermal, or geothermal sources, and electricity for photovoltaic solar, wind, tidal and similar renewable sources. This is a logical boundary as it is the first instance where the energy in nature is converted into a form within human influence.

\section{E. Importance of primary energy}

Attention should be given to primary energy demands as it reflects the ultimate energy footprint of the fuel/energy supply chain that is used to power the particular mode of transportation. The primary energy consumption reflects the amount of source energy that the world is drawing down on in the case of on-renewable fossil fuels, once it is used, it cannot be replaced in a timeframe that is meaningful to human civilization. Whilst maximising the effective use primary energy of fossil fuels is a necessary way of combating climate change, a better metric would be carbon dioxide intensity per unit of energy, $\mathrm{CO} 2$ intensity.

In the case of renewable energy, primary energy consumption reflects the capacity that is being used, and how much more is available. As the world transitions to renewable energy [5], the effective use of new energy sources and energy supply chains that minimise losses and maximise efficacy of the system should be considered.

The sun-to-heel metric is useful in this case by placing a number on the true energy cost of our transportation choices. By viewing the primary energy costs instead of the energy consumed by the end user, the implications energy use in transportation can be accounted for past state or national boundaries, to the ultimate shared resource, the earth. Taking a view of the system, on top of individual components, with the entire life cycle of the energy of the trip accounted for, a more complete result and interpretation of life cycle analysis can be obtained.

\section{Methodology}

\section{A. Scope and boundaries}

The sun-to-heel metric seeks to capture the true cost of energy for moving people, as close as possible to the sun that is the source of all our power. This accounting methodology thus begins from the "heel" i.e. the passenger and traces the energy along its supply chain pathways back towards its source, which in this case is the primary energy.

This metric will reflect the amount of primary energy needed, on a per capita basis, needed to move the average commuter on a journey. There is no prejudice on who the person is, or to what intent, if it is occupational or recreational, of commuting. Additionally, the focus is on the human commuter. Freight is excluded from this measurement. The measure is normalised to a per capita basis to negate the effects of city size as larger, more populous cities naturally will consume more resources, not just energy.

\section{B. Energy costs of capital}

Energy used to build and maintain the infrastructure is not included in the sun-to-heel metric. Life cycle analysis can be done to calculate the primary energy costs per trip contributed by capital, infrastructure, roads, rolling stock and others. The life cycle analysis is common study done in the literature to understand the impacts of a good or service through-out its life cycle[6], and is largely what inspired the sun-to-heel metric. Capital energy costs commonly used in the literature is not considered in this paper as $\mathrm{StH}$ only deals with the fuel and energy use that is related directly to mobility [7].

\section{Sun and Heel}

The sun is the source of all energy on Earth, eventually. Fossil fuels, coal, oil, and natural gas, are made up of the remains of plants and animals that lived millions of years ago and buried under tonnes of rock through the geological process. While they were alive, these plants got their energy from photosynthesis of light from the sun, the sun's stored energy in plants is transferred to animals when consumed by them. Wind energy originates from the unequal heating of the Earth's surface by the sun, along with the Earth's rotation. 
Solar energy, whether photovoltaic or thermal, is derived directly from the sun.

The boundary this metric uses for measuring the efficiency of conversions between different forms of energy, namely light, chemical, mechanical begins at the point where humans first take possession of the energy, even though it is not the amount of energy entering the Earth from the sun, meaning the name of this metric might be taken as a bit of a misnomer. Complete information on the conversion pathways of energy from the moment it enters earth, for example solar heating of the earth surface, leading to air pressure differences, which results in moving air, is beyond current human knowledge, and not necessary to fulfil our purpose of energy efficiency.

The focus of the sun-to-heel matric is that of energy efficiency, not greenhouse gas emissions as the intention in this paper is not to provide any sort of yardstick for carbon efficiency or carbon intensity but to adopt a wider view to understand the trade-offs, if any, between moving from a carbon intensive form of fuel, to a decarbonised future. This metric on system-wide energy efficiency will provide insights that will continue to be relevant in a highly electrified, post carbon world.

\section{Sun-to-heel metric}

The sun-to-heel metric for energy efficiency (Joules per trip) is expressed

energy efficiency $=\frac{J}{\text { Trip }}$

where $J$ is the amount of primary energy used (Joules) and Trip is the number of trips.

\section{Definition of the Numerator}

The numerator is amount of primary energy used. This is a move away from traditional measures of energy such as Btu (British Thermal Units) or toe (Tonne of oil equivalent) to International System of Units for energy used in the literature [8]. As the world is transitioning away from petrol or diesel fuelled personal vehicles, several technologies that can potentially replace the internal combustion engine (such as battery electric vehicles (BEV or EVs - electric vehicles) and hydrogen fuel cells) do not need any fossil fuel in their operation or in the energy supply chain leading up to them. As such, the usage of Joules as the unit of measure for energy is more appropriate in response to changes in the technological landscape.

The numerator's measurement in Joules includes the amount of energy needed to move the vehicle and the commuter over the distance of a given trip. Using an example of a commuter journey via car, the numerator in the sun-toheel metric includes the energy needed to move the car and the commuter in their trip and the energy needed to bring the fuel to the car. In short, this is the primary energy required for a trip.

\section{Definition of the Denominator}

A key difference between the transportation energy efficiency measurement defined in this paper and others in the literature [9] is that distance is not found in the denominator. Energy efficiency metrices that is fuel usage per unit distance based (such as mpg (miles per gallon), or $1 / 100 \mathrm{~km}$ (litres per 100 kilometres)) measures fuel efficiency by using a ratio between the volume of fuel used and the distance travelled. These two matrices apply chiefly to fossil fuel fuelled vehicles, reflecting the fuel economy of the vehicle. The better the fuel economy, the further the vehicle can travel on a given amount of fuel. Such a metric is useful for consumers to make informed purchasing decisions on the total cost of ownership of the vehicle, and for the environmentally conscious, insight into the environmental cost of using the vehicle. However, picking out a car that has the best fuel economy is based on a complex question that the better fuel economy, ceteris paribus, all things equal, is better for the environment, and a lower cost of operating the vehicle and thus ownership which unfortunately is not true. The economic and environmental costs also depend on the amount the vehicle is used i.e. the further the distance travelled, the greater the amount of fuel consumed and the higher the costs, regardless of the fuel efficiency of the vehicle. Moreover, the miles per gallon and litres per $100 \mathrm{~km}$ metrics assume that the internal combustion engine driven vehicles are the primary means of providing for commuting services which may not be the case for modern cities where many other options are available.

Energy used per passenger kilometre is a similar to the per unit distance based metrics but is usually used for trains or buses and suffers from the same problem as fuel usage per unit distance metrics.

In modern civilization, the distance we need to travel is a variable that we have influence over. For example, by living closer to the workplace, one can reduce the distance of the daily commute. Fuel efficiency per distances metrics is useful for comparing vehicles and pushing for improvements in their fuel economy. However, a higher level metric which takes the perspective of the commute as the ultimate goal, and normalises to it, will provide a system view on energy efficiency. In this paper, the number of trips for the amount of energy used is proposed as a denominator and the true cost of a commute can be reflected, be it a case by case basis, or a per capita basis. Energy costs, environmental costs can be seen through the lens of commuting as a service provided to the commuter.

\section{E. Definition of an average trip}

The definition of an average trip is the distance travelled and time taken for a typical trip in given city by a resident in the city. It should be indicative of a trip that the resident of the city makes regularly and routinely for their economic activities. The intent of this paper is to measure the efficiency of a city's various modes of transportation in supporting the city's economic activity and taking the trips most commonly made would exclude exceptional events, such as major sporting events or on days of major road closures due to security reasons.

In a modern city, residents have a choice of various modes of transportation and often different types of modes work together to provide door-to-door transportation solution across different modes and networks. The most rudimentary model would be the door-to-door commute by private or hired car. The commuter walks from his home, to the vehicle, then drives or is driven to the destination, the commuter then walks the remaining distance to his workplace, the destination 'door'. Another combination of modes, using a typical of 
public transportation, would be a walk-bus-walk-train-walk, where the commuter walks from their home to take a bus to a metro station, after the subway ride, walks the "last mile" to the workplace. Other permutations are possible, for example, the walking segments can be replaced by bicycle rides, personal mobility devices, the subway ride can be replaced by a longer bus ride. The whole commute could be done by a single mode of transportation, for example by only using the subway. The model may be applied on the personal level to provide a personalised assessment of the primary energy impact by one's commute or it can be used as a city-wide assessment, either as an accounting of total primary energy consumed by the city for transportation, broken down by the various modes. It can also be used within a city, for comparison between different options of commute, for example between private car transportation, and public transport.

\section{F. Permutations of modes of travel}

In modern cities, there are usually many options of transportation. A commuter that wants to get from point A to point $\mathrm{B}$ has multiple options to choose from, be it by private or public transport. In this example, a commuter traveling from origin A to destination B has three options as shown in Table I.

- Option 1: Drive from origin A to destination B with a walking to and from the car.

- Option 2: Using public transport using the city's network of bus and trains.

- Option 3: The commuter may take the train, but instead of taking the bus to the train station, they can use a bike sharing system and cycle to the station instead, and cycle from the train station to the Destination B.

TABLE I

Permutations of Modes of TRAVEL

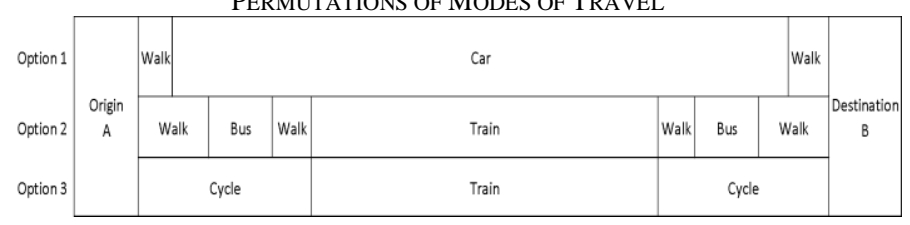

In the three options presented, the point origin $\mathrm{A}$ and destination B are the same geographical points for the three options. The difference between the three options is the modes of transportation and as a result, the specific energy per passenger distance will vary and the amount of primary energy used will be different. The distance between different permutations of modes of transportations is expected to be different even when the destination and origin locations are the same. This is because the routes travelled are different, for instance a more direct route may be taken if a private car is used, as compared to a bus-train mode where the commuter might may not be able to travel the route of their choice to the destination - having to follow the route the public bus or train takes, which usually results in a detour. The total energy consumed for a trip is therefore the sum of the energy used in each of the stages for an option. For example, the total primary energy requirement for Option 3 would be the sum of the primary energy required for cycling to the train station, the primary energy used to move that commuter through the network, and the primary energy needed for the commuter to cycle from the train station to the destination. The primary energy used at each stage is the amount of energy consumed at point of use i.e. by a car or by a person walking, divided by the efficiency of the supply chain bringing energy to the point of use.

\section{G. Energy consumed at point of use}

The amount of primary energy used, $J$, per trip is the sum of the primary energy required at each of the stages in transportation as described in Equation (1). The product of the amount of energy consumed by the mode of energy for the trip, $C$, multiplied by the energy efficiency of the entire energy supply chain that feeds into that stage transportation, $E$. Therefore, the primary energy required for a trip is the sum of all the products $C_{i} E_{i}$ to $C_{n} E_{n}$ where $i$ to $n$ are the stages in the trip.

$$
\text { energy efficiency }=\frac{\sum_{n}^{i}\left[C_{i} E_{i} \ldots C_{n} E_{n}\right]}{\operatorname{Trip}}
$$

The energy consumed by the vehicles, $C$, to move one commuter through the length of the trip is the specific energy needed per passenger kilometre multiplied by the distance of the trip. This is essentially the fuel/energy consumption for the trip. For example if a stage of the trip is made by car, and 1 litre of petrol is used, then $C$ for this trip would be the energy contained in 1 litre of petrol divided by the number of people who were transported by the car(note that if the car were a taxi, the driver does not count to the number of people transported). Thus the formula for $C$ is:

$$
C=\text { specific energy per passenger } \times \text { distance of stage }
$$

$C=\frac{\text { energy per } \mathrm{km} \text { of mode of transport } \times \text { distance of stage }}{\text { number of people transported }}$

(4)

Specific energy per passenger means the energy required to move one person over a unit distance. Using a train as an example, it would be the amount of energy required to move the whole train with its loading of say 800 people over a distance of $1 \mathrm{~km}$, divided by the number of people the train is carrying. The actual average loading should be used in this case and not the theoretical maximum carrying capacity of the vehicle. For private cars, although most cars are designed to carry five or more people (typically one driver and 4 passengers), the car itself is rarely loaded to its full capacity in daily commute. Thus, the loading factor used for cars will not be 5, but around 1.7 passengers for cities such as Singapore [10]. Note that a key difference between the sun-to-heel metric and fuel economic metrices like miles per gallon is that the distance variable has been effectively moved from the denominator to the numerator which means that an energy efficient trip is not just one that uses a fuel efficient vehicle, but also one that minimises the distance that needs to be covered.

The efficiency of the energy supply chain, $E$, is the cumulative efficiency of the entire supply chain that extracts and delivers energy from the primary energy source to the vehicle. This is the well-to-tank portion of the well-to-wheels model [11]. Using the petrol car as an example, this would be the ratio of the amount of energy contained in one unit of 
petrol, and the amount of energy contained in the crude oil that was the raw material for that unit of petrol. This is to account for the energy that is needed to extract, refine, transport, the fuel before reaches the vehicle. In the case of electric modes of transportation, for example the electric train, the energy supply chain will consist of, for example, extraction of fossil fuels, refinement, thermal generation of electricity and transmission.

$$
E_{n}=e_{n a} \cdot e_{n b} \ldots e_{n z}
$$

Thus, the efficiency of the energy supply chain supplying the vehicle in $C_{n}$ is the product of all the stages of energy conversion from the point of extraction, the point where the energy passes into human influence, to the point where it is stored or transferred onboard the vehicle.

Below are two simplified examples typical energy supply chains from a primary source to a point of use. The first example shows a typical supply chain of a fuels used in internal combustion engines, gasoline or diesel for example. The chain begins with the crude oil in the ground, which is then extracted, refined at refineries, transported to and distributed in cities. At each step of the supply chain, energy is either needed as an input, or is lost through other means. In other words, at each step of the supply chain, more energy enters the system represented by the step than leaves it, thus the system is less than $100 \%$ efficient.

Similarly, the energy supply chain for non-fossil fuels can be represented. As the boundary for this model starts at the point where energy first enters human influence, the energy for photo-voltaic solar power begins from the output of the solar panels, meaning that the conversion efficiency of solar irradiance to electricity is beyond the scope of this model. In the case for solar PV, the example below assumes electricity from solar panels are directly used an electrical vehicle or train. Table II shows some examples where detailed modelling of actual circumstances can become much more complicated.

TABLE II

PERMUTATIONS ON ENERGY SUPPLy CHAIN

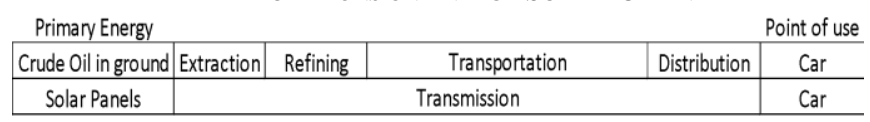

\begin{tabular}{|c|c|c|c|c|}
\hline Primary Energy & & & & Point of use \\
\hline Crude Oil in ground & Extraction & Refining & \begin{tabular}{|l|l|l} 
Transportation & Power generation & Transmission \\
\end{tabular} & Train \\
\hline Solar Panels & & & Transmission & Train \\
\hline
\end{tabular}

The energy efficiency of the vehicle, meaning the efficiency of the vehicle to convert the energy stored in the fuel to kinetic energy, is not part of the formula as its impacts are already included in the energy used by the mode of transportation. Ceteris paribus, a more fuel inefficient vehicle will use more energy (i.e. higher specific energy per passenger, thus higher $C$ above)

$C . E=\frac{\text { (Specific energy per } k m \times \text { distance traveled) }}{e_{n a} \cdot e_{n b} \cdot e_{n c} \ldots e_{n z}}$

The full formula for the sun-to-heel metric is:

$$
\begin{gathered}
\begin{array}{c}
\text { sun }- \text { to }- \text { heel energy efficiency } \\
\sum_{n}^{i} \frac{(\text { Specific energy per } \mathrm{km} \times \text { distance traveled })}{e_{n a} \cdot e_{n b} \cdot e_{n c} \ldots e_{n z}}
\end{array} \\
\text { Trip }
\end{gathered}
$$

The sun-to-heel energy efficiency metric is a function of the summation of the specific energy per distance of the vehicles, the distance travelled per trip, and the efficiency of the energy supply chain. This provides a basis for comparison of energy usage between different modes of transportation when getting from an origin and destination. It can also be used to measure the performance of a city or between cities.

\section{H. Walking}

Walking is not a usual part of transportation analysis, especially when it comes to energy use. Calories burned by human beings is not normally thought of as energy consumed, understandably so as much of the world's population has excess energy to burn [12]. It is conceivable to think of the energy consumed during transportation to be small, however, getting a joule of energy to a human being may cost as much as 35 times as much energy [13] can create a significant multiplier effect.

The distance covered by walking in our models is expected to be small compared to other modes of transportation. Commuters do not generally choose to walk long distances, the distance covered by the scenarios will likely be the minimum distance the commuter has no choice but to cover, for example, from the home to the nearest bus stop or subway station; or even a dozen or so meters from home to the car park. Another scenario that could be a simulated walking only scenario such as a what-if scenario of an extreme "health nut" where the commuter walks, without the use of any vehicles, private or public, or even bicycles, to work. This is not only an interesting academic question, but to address the underlying assumption generally made in transportation studies, that if a commuter walks, or rather does not take a form of transportation, his or her impacts and resource requirements drops to zero. Factoring in walking will prevent this form of energy expenditure from becoming unaccounted for. Moreover, in urban fabrics that focus on TOD (Transit Oriented Design), the proportion of time and distance covered by walking be greater versus other modes of transportation. If energy consumed due to walking is not taken into consideration, a significant portion of energy used during transportation may be unaccounted for.

\section{CONCLUSION}

Current metrics for energy efficiency in transportation focuses on the efficiency of the vehicle used in transportation. While such matrices have set the foundations for measuring energy efficiency, there is a need to advance performance matrices to keep up with the coming changes in the global energy economy, the rapidly urbanising landscape, and the need for environmental stewardship. This paper has proposed the use of the sun-to-heel metric for life cycle analysis of energy efficiency of transportation fuels as opposed to the conventional oil-to-wheel method of analysis. It is demonstrated that the proposed method is capable to provide a holistic system wide perspective based on trips, including first and last mile connections are considered during the environmental impact assessment of a transport product or service. While the Sun-to-heel metric provides insights into the true cost of primary energy of transportation, it does not include a crucial component of commuting time. Nevertheless, 
the metric in its current form provides a means of measuring the trade-offs between different modes of transportations with respects to energy consumption

\section{REFERENCES}

[1] Shell, Sky, "Meeting the goals of the Paris agreement", 2018.

[2] Argonne National Laboratory, Argonne's GREET Model , 2018.

[3] T. Atkins, M. Escudier, "A dictionary of mechanical engineering", 2013.

[4] Eurostat, "Calculation methodologies for the share of renewables in energy consumption - Statistics Explained," 2018.

[5] OECD \& IEA, "Market Report Series: Renewables 2017, analysis and forecasts to 2022," 104-42, 20142, 2017.

[6] International Standards Organisation, "ISO14040:2006 Environmental Management - Life cycle assessment Principles and framework," 2006.

[7] V. Fthenakis, and H. Kim, "Solar Energy, Photovoltaics: Life-cycle analyses," 85-8, 1609-1628, 2011.

[8] N. Risk and F. Collaboration, "The Lancet," 387-10026, 1377-1396 , 2014.

[9] Oxford, “A Dictionary of Chemical Engineering," 2014

[10] Argonne National Laboratory, Argonne's GREET Model, 2018.

[11] DBS, "Reinventing the Wheel," 13, 2017.

[12] W. Shen, W. Han, D. Chock, Q. Chai, and A. Zhang, "Well-to-wheels life-cycle analysis of alternative fuels and vehicle technologies in China,"Energy Policy, 49, 296-307, 2012.

[13] M. Verma, "Energy Use in Global Food Production Considerations for Sustainable Food Security in the $21^{\text {st }}$ Century," Springer briefs in food, health, and nutrition, 2015. 